\title{
BMJ Open Frailty measurement and outcomes in interventional studies: protocol for a systematic review of randomised control trials
}

\author{
Melissa Shears, ${ }^{1}$ Danielle McGolrick, ${ }^{2}$ Braden Waters, ${ }^{3}$ Marnie Jakab, ${ }^{1}$ \\ J. Gordon Boyd, ${ }^{2}$ John Muscedere ${ }^{2}$
}

To cite: Shears M, McGolrick D, Waters B, et al. Frailty measurement and outcomes in interventional studies: protocol for a systematic review of randomised control trials. BMJ Open 2017;7:e018872. doi:10.1136/ bmjopen-2017-018872

- Prepublication history and additional material for this paper are available online. To view these files, please visit the journal online (http://dx.doi. org/10.1136/bmjopen-2017018872).

Received 27 July 2017 Revised 20 0ctober 2017 Accepted 17 November 2017

\section{CrossMark}

${ }^{1}$ School of Medicine, Queen's University, Kingston, Ontario, Canada

${ }^{2}$ Department of Critical Care Medicine, Queen's University, Kingston, Ontario, Canada

${ }^{3}$ Division of Critical Care

Medicine, McMaster University, Hamilton, Ontario, Canada

Correspondence to

Dr John Muscedere; muscedej@kgh.kari.net

\section{ABSTRACT}

Introduction Frailty is associated with reduced functional capacity, decreased resistance to stressors and is predictive of a range of adverse health outcomes, including dependency, hospitalisation and mortality. Early identification of frailty may prevent, reduce and postpone adverse health outcomes. However, there is a need for additional evidence to guide decision-making for the care of frail patients since frail persons are frequently excluded from studies, the differential impact of frailty is often not examined in clinical trials and few large-scale clinical trials examining frail cohorts have been conducted. Randomised control trials (RCTs) published to date have used a diverse range of definitions of frailty, as well as a variety of outcome measures. The objective of this systematic review is to comprehensively characterise the frail populations enrolled and the end points reported in frailty RCTs. Methods and analysis We will identify all RCTs reporting on the outcome of interventions in adult (age $\geq 18$ years) frail populations as defined by authors, in all settings of care. Databases will include MEDLINE, CINAHL, EMBASE, Psyclnfo, Global Health, the Joanna Briggs database and Cochrane Library. Two reviewers will independently determine trial eligibility. For each included trial, we will conduct duplicate independent data extraction, interrater reliability, risk of bias assessment and evaluation of the quality of the evidence using the Grading of Recommendations, Assessment, Development and Evaluations approach.

Ethics and dissemination This systematic review will comprehensively identify RCTs including frail patients to identify how frailty is measured and which outcomes are reported. The results of this systematic review may inform clinicians caring for persons with frailty, facilitate conduct of future RCTs and inform future efforts to develop common data elements and core outcomes for frailty studies. Our findings will be disseminated through conference presentation and publication in peer-reviewed journals.

PROSPERO registration number CRD42017065233.

\section{INTRODUCTION}

Frailty is a state of increased vulnerability, associated with loss of functional capacity and reduced ability to cope with normal or
Strengths and limitations of this study

- This is a comprehensive systematic review of randomised control trials studying the impact of an intervention in a frail population.

- Other strengths include the use of a comprehensive search strategy of literature published in peerreviewed journals, detailed inclusion and exclusion criteria, duplicate independent screening and data extraction, and the conduct of independent duplicate risk of bias assessment and assessment of the quality of evidence using the Grading of Recommendations, Assessment, Development and Evaluations approach.

- Conclusions of this study will be limited to the number and quality of trials conducted.

- The differences in populations included, interventions and outcome measures used may be a source of heterogeneity among studies evaluated.

- This systematic review will inform the development of common data elements and core outcomes for future trials studying the impact of interventions on frail patients.

disease-based stressors. ${ }^{12}$ As a multidimensional concept, frailty is associated with a range of adverse health outcomes, including dependency, hospitalisation and mortality. ${ }^{3-6}$ From a healthcare perspective, the early identification of frailty is important as this may prevent, reduce or postpone adverse health outcomes for adults living with frailty. Based on an European cohort study and two systematic reviews involving community-dwelling older adults, the reported prevalence of frailty ranges from $10 \%$ to $19 \% .^{7-9}$ However, prevalence rates vary based on the populations studied, survey methodology employed and the instruments used to measure frailty. ${ }^{10}$ Even in populations which are often thought of as being mostly frail such as long-term care homes, the prevalence as well as the severity of frailty may vary widely. ${ }^{11}$ Overall 
frailty is common, but there is an increasing need for standardised approaches for the detection and measurement of frailty.

In contrast to the high prevalence of frailty described in many populations, there is a lack of high-quality evidence to guide decision-making for the care of frail patients. ${ }^{12}$ Clinical trials that enrol older adults rarely consider the impact of frailty, as patients living with frailty and/or those with significant comorbidities are often excluded from trials. ${ }^{13}$ Further, there have been few large-scale clinical trials specifically including frail patients. ${ }^{14}$ As a result, current treatments and practice guidelines are based on evidence that may not be applicable to frail patients. Treatments that are effective in non-frail patients are often applied to those with frailty, but they may not be effective and result in harm or wasted resources. As an example, in patients with advanced frailty nearing end of life, invasive and expensive technologies are often aggressively used without improvement in outcomes and worsen quality of life (QoL). ${ }^{17}{ }^{17}$ Conversely, treatments ineffective in fit patients may be effective in those who are frail. ${ }^{18}$ To guide evidence-based practice, there is a need for data that are interpretable, generalisable and applicable to the patients living with frailty encountered by clinicians and decision-makers in practice.

Although there has been an exponential increase in frailty publications and a growing interest in the field, most publications have used observational designs. Furthermore, randomised controlled trials (RCTs) enrolling patients living with frailty have reported a diverse range of definitions of frailty, as well as a variety of outcome measures. ${ }^{14}{ }^{15}$ Importantly, these studies rarely report on patient-centred outcomes, such as QoL. In addition, current studies infrequently determine the degree or severity of frailty. This is problematic, as the severity of frailty correlates with worsened outcomes including increased mortality and institutionalisation. ${ }^{19}$ Variability in inclusion criteria and outcome measures in frailty interventional trials creates a challenge for their interpretation and generalisation, confounding the utilisation of these studies in clinical decision-making. To guide the conduct of future interventional studies of frailty and increase their ability to guide practice, it is necessary to establish a set of common data elements and standardisation of reported outcomes. This has been done in other fields of medicine and given the nascent stage of frailty interventional research, this is urgently required. ${ }^{20}{ }^{21} \mathrm{~A}$ systematic review of the current state of evidence is necessary to inform the future development of common data elements and core outcomes. We hypothesise that there is a wide variety of inclusion criteria, frailty measurements and outcomes reported in trials studying the effects of an intervention in a frail population.

The objectives for this systematic review are: (1) to report on the characterisation of frail populations enrolled in frailty RCTs including the measurement of frailty and (2) to describe the outcomes reported in RCTs addressing frailty.

\section{METHODS AND ANALYSIS}

This systematic review will be conducted according to the Preferred Reporting Items for Systematic Reviews and Meta-Analyses (PRISMA) criteria. $^{22}$ The review protocol will be reported in accordance with the PRISMA-Protocol (PRISMA-P), ${ }^{23}$ and has been registered in PROSPERO on CRD42017065233. Any amendments to this protocol will be updated on PROSPERO and documented accordingly.

\section{Criteria for selecting trials for this review \\ Types of trials}

We will include RCTs of any design (both individual randomisation and cluster randomisation) studying the impact of a patient-level or system-level intervention in a population living with frailty. Only the primary report of interventional trials will be included. Language restrictions will not be imposed and non-English manuscripts will be translated.

We will exclude non-randomised studies, pilot trials that only report feasibility criteria, abstracts, non-peer-reviewed publications and grey literature. We will also exclude planned or ongoing trials found in clinical trial registries and publications reporting on secondary analyses of data. Narrative and systematic reviews will be excluded, but references will be reviewed for possible trials meeting inclusion criteria.

\section{Types of participants}

RCTs meeting inclusion criteria must study the impact of an intervention in adults ( $\geq 18$ years of age) living with frailty as determined by the research question, hypothesis, objectives and/or study inclusion criteria. Any characterisation of frailty as defined by the investigators in each trial will be eligible. Studies that do not include or aim to target a frail population will be excluded. We will exclude studies in which the study population has a high prevalence of frailty but whose objective is not to explicitly study the impact of an intervention on frail patients. We will exclude studies targeting prefrail patients due to the variability as to how this is defined and will exclude publications who study the effect of an intervention on caregivers.

\section{Types of interventions}

This review will include all types of interventions in all settings of care. Trials that study both patient-level and system-level interventions will be included. Exercise/nutrition related interventions, programmes of care, pharmaceutical interventions, home visit interventions, remote healthcare support, community programmes, and self-management programmes are all examples of interventions that may be included for this review.

\section{Types of outcome measures}

Trials that report any outcome will be included; both the primary and secondary outcomes of each trial as categorised by the authors will be abstracted. We will 
abstract both the types of outcomes reported and the impact of the intervention on the outcome. Examples include frailty status, physical function, health service utilisation, muscular strength, change in activities of daily living, QoL, number of falls, bone density, mortality, hospital length of stay and measures of depression.

\section{Subgroup analyses}

We will report on the following subgroups of studies; study quality (low, high), study setting (acute care, longterm care or residential care, primary care), type of frailty measurement used on enrolment (no measurement of frailty, type of validated measurement scale), type of outcome, date of publication (before and including 2010, after 2010) and surgical versus non-surgical populations.

\section{Search methods for identification of trials}

The literature search will be conducted using a two-step strategy designed to maximise retrieval (online supplementary appendix 1). An initial search of MEDLINE will be performed using the Ovid platform. Results from this search will be reviewed by the lead researcher and team for recall. Revisions will be made as necessary, prior to expanding the search. We will search: MEDLINE (1946present), EMBASE (1947-present), PsycInfo (1806present), Global Health and the Joanna Briggs database (1973-present) (all on the Ovid platform), CINAHL (ebsco platform) and the Cochrane Library (Wiley platform).

\section{Trial records}

After identification of potentially relevant trials, all titles and abstracts will be screened independently by reviewers for relevance using the eligibility criteria listed above. This will be conducted in duplicate by the combination of six reviewers using an electronic systematic review software (Covidence.org). ${ }^{24}$ Titles can be marked as yes/no/ maybe. Any titles and abstracts with the combination of 'yes/yes', and 'yes/maybe' will be advanced for full-text review. Titles and abstracts with the combination of 'yes/ no' and 'no/maybe' will be identified as a conflict and resolved by the lead author (JM).

\section{Data collection}

Data will be extracted independently by reviewers, using a data abstraction form (online supplementary appendix 2) that has been developed and piloted on 10 randomly selected included studies. The data abstraction form will be reviewed for any discrepancies, which will secondarily serve as a calibration exercise. The form will be refined accordingly prior to proceeding with data abstraction. The data that will be abstracted will include title, first author, year of publication, study design, number of centres, definition of frailty, relevant baseline patient data, intervention and comparator, setting of care, primary outcome (definition and results), secondary outcomes (definitions and results), duration of follow-up and QoL data. Disagreements will be settled by consensus and adjudication by the lead author (JM). Inter-rater reliability between reviewers will be established using a к score.

\section{Assessment of risk bias}

Trial methodology will be evaluated using a modified Cochrane Collaboration tool for assessing risk of bias. ${ }^{25} 26$ The primary outcome for each included RCT will receive a description and judgement of 'low risk', 'unclear risk' and 'high risk'. Two reviewers will independently perform the risk of bias assessment, with disagreements resolved by discussion. We will consider the risk of bias as 'high' when bias is both present and likely to affect outcomes, and 'low' when the bias is not present, or present but unlikely to affect outcomes.

\section{Summarising data and treatment effect}

The outcome data gathered will be presented in tables, as well as with an accompanying narrative analysis of these data where applicable. In addition, a meta-analysis of primary and secondary outcomes will be conducted where possible. Binary outcomes will be analysed using Mantel-Haenszel statistical methods, random effects models and reported as risk ratios with 95\% CI. Continuous outcomes will be pooled using the inverse variance method and random effects model to determine treatment effect and reported as the mean differences with $95 \%$ CI. Meta-analyses will be presented as forest plots, where applicable. Primary authors will not be contacted regarding missing or clarification of data. All analyses will be performed using RevMan software V.5.3 (Review Manager, the Nordic Cochrane Centre, the Cochrane Collaboration 2014 , Copenhagen, Denmark).

\section{Assessment of heterogeneity}

Heterogeneity will be determined for each outcome using the $\chi^{2}$ test and intraclass correlation $\left(\mathrm{I}^{2}\right)$.

\section{Assessment of reporting bias}

We will investigate the possibility of publication bias using a funnel plot, provided there are at least 10 included studies. ${ }^{27}$ Risk of bias will be assessed by visual inspection of a funnel plot constructed by plotting effect size versus SE.

\section{Assessment of confidence in estimates of effect}

We will assess the quality of evidence for the interventions, examining each outcome using the Grading of Recommendations, Assessment, Development and Evaluations approach and rating system. ${ }^{28}$ RCTs will start as high-quality evidence but may be rated down by one of the following: risk of bias, imprecision, inconsistency, indirectness and publication bias. Quality of evidence will be assessed by two independent reviewers. Disagreements will be resolved by consensus. 


\section{DISCUSSION}

Frailty describes a clinical state of increased vulnerability to external stressors and decreased functional capacity. Frailty is quantifiable and higher severity of frailty is associated with increased mortality, morbidity and institutionalisation. ${ }^{19}$ There have been increasing efforts to study interventions aimed at improving outcomes for patients living with frailty with randomised controlled studies. However, these studies have used various frailty measurement tools and have reported on a wide range of outcome measures. These inconsistencies limit our ability to translate results of intervention-based RCTs into clinical practice. This systematic review will employ rigorous methodology to summarise the existing data on interventions for patients living with frailty. Our main objective is to describe the characterisation and measurement of frailty along with the outcomes and end points used in the interventional trials reported to date. Further, this review will enhance our understanding of described interventions for this growing and vulnerable patient population.

A limitation of this study is the inclusion of studies over a wide span of time and the evolution in our understanding of frailty over that time. In spite of this limitation, it is important to understand trial evidence in its entirety, therefore we have included all published frailty trials. We will mitigate this limitation by also reporting on a subgroup of more recent trials published after 2010. In addition, due to the large scope of this review, we have not included secondary publications of trials which may result in the under-reporting of some outcomes including economic analyses which are commonly not described in primary publications.

In conclusion, this review will serve to guide future initiatives aimed at developing standardised core data elements, data dictionaries and outcomes in interventional frailty studies. Ultimately this will facilitate the analysis, interpretation, generalisability and aggregation of future frailty RCTs and hence their ability to guide clinical practice and care for populations living with frailty.

Acknowledgements The authors thank Amanda Ross-White, a Health Sciences Librarian at Queen's University, for conducting the literature search.

Contributors JM conceived the idea for this systematic review. JM, MS and DM developed the methodology for the systematic review protocol. The manuscript was drafted by MS and revised by DM, BW, MJ, JGB and JM. JM and DM developed the search strategy. MS, DM, BW, MJ, JGB and JM will screen potential studies and MS and DM will perform full text review. Conflicts will be resolved by JM. MS, DM, BW, MJ, JGB and JM will perform duplicate independent data extraction, risk of bias assessment, GRADE assessment and data synthesis. JM is the guarantor of the review.

Funding This study was facilitated and funded by the Canadian Frailty Network (CFN) which is a non-for-profit organisation funded by the government of Canada through the Networks of Centres of Excellence (NCE) programme. CFN had no role in the conduct, analysis or reporting of the study.

Competing interests JM is the Scientific Director for the Canadian Frailty Network. Provenance and peer review Not commissioned; externally peer reviewed.

Open Access This is an Open Access article distributed in accordance with the Creative Commons Attribution Non Commercial (CC BY-NC 4.0) license, which permits others to distribute, remix, adapt, build upon this work non-commercially, and license their derivative works on different terms, provided the original work is properly cited and the use is non-commercial. See: http://creativecommons.org/ licenses/by-nc/4.0/

(C) Article author(s) (or their employer(s) unless otherwise stated in the text of the article) 2017. All rights reserved. No commercial use is permitted unless otherwise expressly granted.

\section{REFERENCES}

1. Viña J, Tarazona-Santabalbina FJ, Pérez-Ros P, et al. Biology of frailty: modulation of ageing genes and its importance to prevent age-associated loss of function. Mol Aspects Med 2016;50:88-108.

2. Clegg A, Young J, lliffe S, et al. Frailty in elderly people. Lancet 2013;381:752-62.

3. Fried LP, Ferrucci L, Darer J, et al. Untangling the concepts of disability, frailty, and comorbidity: implications for improved targeting and care. J Gerontol A Biol Sci Med Sci 2004:59:M255-63.

4. Fried LP, Tangen CM, Walston J, et al. Frailty in older adults: evidence for a phenotype. J Gerontol A Biol Sci Med Sci 2001;56:M146-57.

5. Campbell AJ, Buchner DM. Unstable disability and the fluctuations of frailty. Age Ageing 1997;26:315-8.

6. Rockwood K, Fox RA, Stolee P, et al. Frailty in elderly people: an evolving concept. CMAJ 1994;150:489-95.

7. Santos-Eggimann B, Cuénoud P, Spagnoli J, et al. Prevalence of frailty in middle-aged and older community-dwelling Europeans living in 10 countries. J Gerontol A Biol Sci Med Sci 2009;64:675-81.

8. Collard RM, Boter $\mathrm{H}$, Schoevers RA, et al. Prevalence of frailty in community-dwelling older persons: a systematic review. J Am Geriatr Soc 2012;60:1487-92.

9. Verlaan S, Ligthart-Melis GC, Wijers SLJ, et al. High prevalence of physical frailty among community-dwelling malnourished older adults-a systematic review and meta-analysis. J Am Med Dir Assoc 2017;18:374-82.

10. Kehler DS, Ferguson T, Stammers AN, et al. Prevalence of frailty in Canadians 18-79 years old in the Canadian Health Measures Survey. BMC Geriatr 2017;17:28.

11. Kojima G. Prevalence of frailty in nursing homes: a systematic review and meta-analysis. J Am Med Dir Assoc 2015;16:940-5.

12. van Weert JC, van Munster BC, Sanders R, et al. Decision aids to help older people make health decisions: a systematic review and meta-analysis. BMC Med Inform Decis Mak 2016;16:45.

13. Zulman DM, Sussman JB, Chen X, et al. Examining the evidence: a systematic review of the inclusion and analysis of older adults in randomized controlled trials. J Gen Intern Med 2011;26:783-90.

14. Bibas L, Levi M, Bendayan M, et al. Therapeutic interventions for frail elderly patients: part I. Published randomized trials. Prog Cardiovasc Dis 2014;57:134-43.

15. Puts MTE, Toubasi S, Andrew MK, et al. Interventions to prevent or reduce the level of frailty in community-dwelling older adults: a scoping review of the literature and international policies. Age Ageing 2017;46:383-92.

16. Kurella Tamura M, Covinsky KE, Chertow GM, et al. Functional status of elderly adults before and after initiation of dialysis. N Engl J Med 2009;361:1539-47.

17. Smith C, Da Silva-Gane M, Chandna S, et al. Choosing not to dialyse: evaluation of planned non-dialytic management in a cohort of patients with end-stage renal failure. Nephron Clin Pract 2003;95:c40-6.

18. Lozano-Montoya I, Correa-Pérez A, Abraha I, et al. Nonpharmacological interventions to treat physical frailty and sarcopenia in older patients: a systematic overview - the SENATOR Project ONTOP Series. Clin Interv Aging 2017;12:721-40.

19. Rockwood K, Song X, MacKnight $\mathrm{C}$, et al. A global clinical measure of fitness and frailty in elderly people. CMAJ 2005;173:489-95.

20. Boers M, Kirwan JR, Wells G, et al. Developing core outcome measurement sets for clinical trials: OMERACT filter 2.0. J Clin Epidemiol 2014;67:745-53.

21. Needham DM, Sepulveda KA, Dinglas VD, et al. Core outcome measures for clinical research in acute respiratory failure survivors. An international modified delphi consensus study. Am J Respir Crit Care Med 2017;196:1122-30.

22. Moher D, Shamseer L, Clarke M, et al. Preferred reporting items for systematic review and meta-analysis protocols (PRISMA-P) 2015 statement. Syst Rev 2015;4:1.

23. Shamseer L, Moher D, Clarke M, et al. Preferred reporting items for systematic review and meta-analysis protocols (PRISMA-P) 2015: elaboration and explanation. BMJ 2015;349:g7647.

24. VH Innovation. Covidence systematic review software. Melbourne, Australia: VH Innovation. 
25. Akl EA, Sun X, Busse JW, et al. Specific instructions for estimating unclearly reported blinding status in randomized trials were reliable and valid. J Clin Epidemiol 2012;65:262-7.

26. Higgins JP, Altman DG, Gøtzsche PC, et al. The Cochrane Collaboration's tool for assessing risk of bias in randomised trials. BMJ 2011;343:d5928.
27. Sterne JA, Sutton AJ, loannidis JP, et al. Recommendations for examining and interpreting funnel plot asymmetry in meta-analyses of randomised controlled trials. BMJ 2011;343:d4002.

28. Guyatt $\mathrm{GH}$, Oxman $\mathrm{AD}$, Schünemann $\mathrm{HJ}$, et al. GRADE quidelines: a new series of articles in the Journal of Clinical Epidemiology. J Clin Epidemiol 2011;64:380-2. 